\title{
THE APPLICATION OF SYSTEMS ENGINEERING PRINCIPLES TO MODEL LITHIUM ION BATTERY VOLTAGE
}

\author{
A Project Report \\ Presented to \\ The Faculty of \\ California Polytechnic State University at San Luis Obispo
}

\author{
In Partial Fulfillment \\ of the Requirements for the Degree \\ Master of Science in Aerospace Engineering
}

By

G. Clayton Gibbs

December 2012 
(C) 2012

G. Clayton Gibbs

\section{ALL RIGHTS RESERVED}




\section{COMMITTEE MEMBERSHIP}

TITLE:

The Application of Systems Engineering Principles to Model Lithium Ion Battery Voltage
AUTHOR:
G. Clayton Gibbs

DATE SUBMITTED:

December 2012

COMMITEE CHAIR: Dr. Jordi Puig-Suari, Professor Aerospace Engineering

COMMITEE MEMBER: $\quad$ Dr. Eric Mehiel, Department Chair, Aerospace Engineering

COMMITTEE MEMBER: $\quad$ Dr. Mark Isaacson, Lockheed Martin Space Systems Company, Battery Engineer 


\section{ABSTRACT \\ The Application of Systems Engineering Principles to Model Lithium Ion Battery Voltage \\ G. Clayton Gibbs}

The objective of this project is to present a Lithium Ion battery voltage model derived using systems engineering principles. This paper will describe the details of the model and the implementation of the model in practical use in a power system. Additionally, the model code is described and results of the model output are compared to battery cell test data. Finally, recommendations for increased model fidelity and capability are summarized.

The modeling theory has been previously documented in the literature but detailed implementation and application of the modeling theory is shown. The detailed battery cell test voltage profiles are proprietary; as such this project will not include axis values, often used in presentation of proprietary data in the public domain. The objective of this presentation is still achieved, as the modeling implementation and results are clearly demonstrated.

Keywords: battery, lithium ion, model, voltage 


\section{Acknowledgments}

I would like to thank the Aerospace Engineering professors at California Polytechnic State University at San Luis Obispo. I would especially like to thank Dr. Eric Mehiel, my project advisor and Dr. Mark Isaacson, my Lockheed Martin Advisor. The support and cooperation of the various programs and personnel at Lockheed Martin that contributed to this study are also gratefully acknowledged. Specifically, Dr. Mark Isaacson and Dr. Patrick Bailey are recognized for their significant contributions to the modeling theory and macro development. 


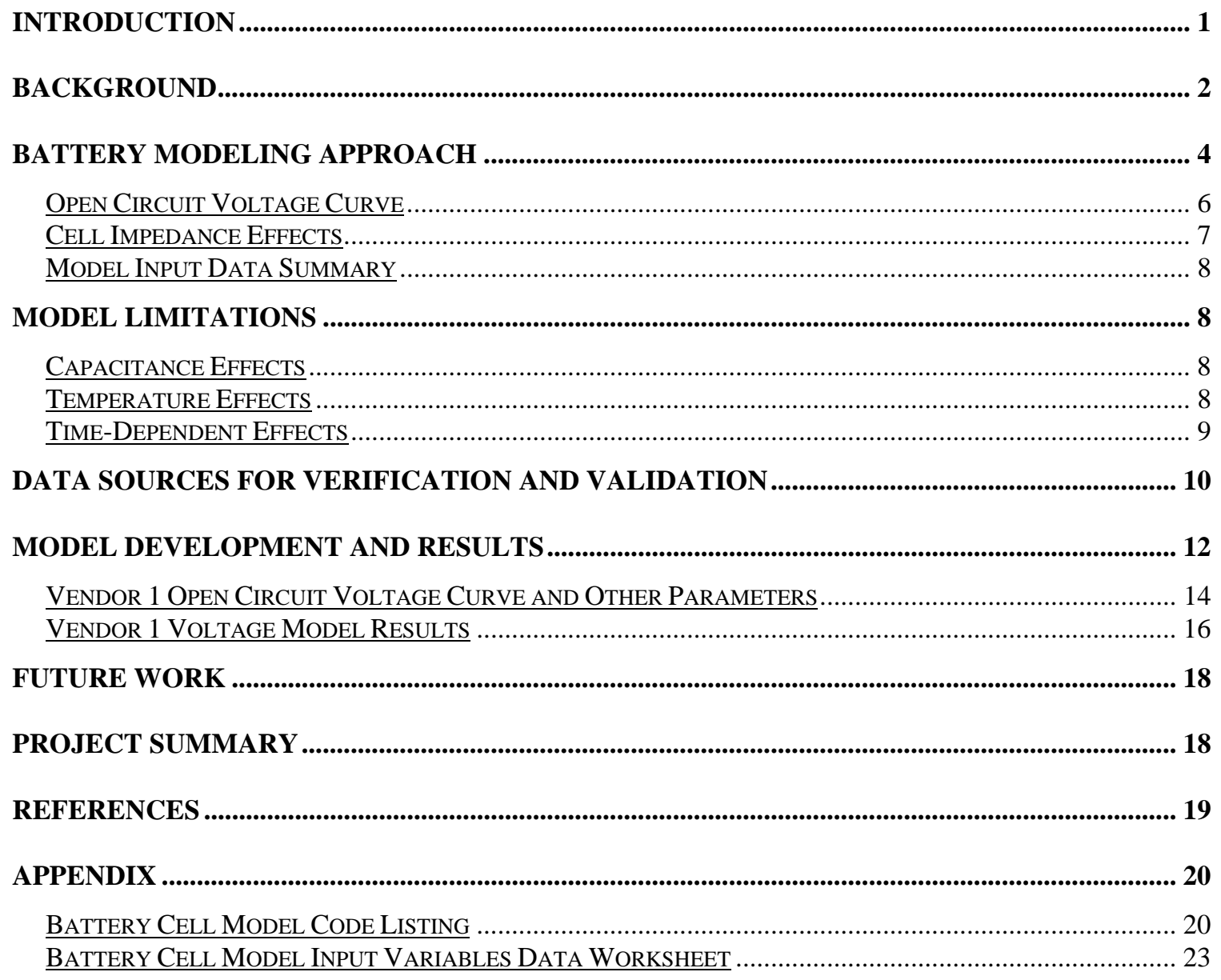




\section{Figure}

\section{List of Figures}

Number

Title

Page No.

FIGURE 1. SAMPLE LI ION MODEL VOCV CURVE.................................................................................. 15

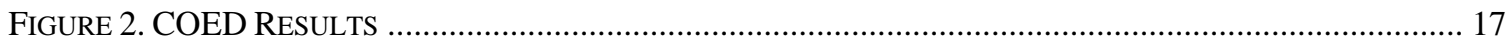

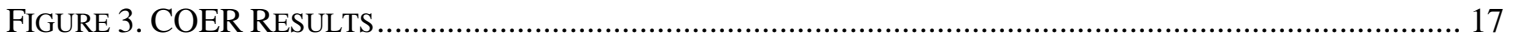

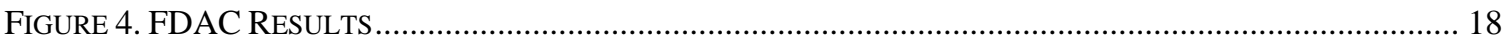

Figure 5. SAmple Battery Cell Model Code InPut VARiable Data SPREAdSheEt ............................ 24 


\section{Introduction}

Batteries have become essential to our everyday life. They are used in many different applications across a variety of industries from health care to consumer electronics to automobiles. They are also used in practically every aerospace application imaginable. Like most technologies, batteries have continued to evolve throughout time, especially for particular applications that have extraordinary requirements. The aerospace industry, with satellite power systems being a prime example, often requires advanced battery technologies for a variety of reasons. Although one reason is often the need for enabling technology, another more common reason is competitiveness; that is, the evolution of the "better, faster, cheaper" technology is necessary for one aerospace company to stay ahead of another, or in some cases to keep up with another. However, the application of a new battery technology to a power system presents new challenges. The system must be capable of dealing with the uniqe properties of the new battery technology and the benefit, or need, of using it must outweigh the performance of the heritage system. Lithium Ion batteries are a relatively new space battery chemistry that have revolutionized new mission capabilities and optimized heritage systems in the aspects of better, faster and cheaper.

A satellite power system is a complicated mixture of components and requires complex modeling to ensure proper sizing and evaluation of all modes of operation. Typically, the power system model is composed of individual component models where applicable. A crucial component model in nearly all power system models is the battery voltage model. High fidelity battery models enhance the power system model fidelity and allow less design margin and higher system efficiency given equal risk. The models 
developed herein are currently in use for systems that require Lithium Ion batteries as an enabling technology.

\section{Background}

This project documents the development of Lithium-Ion (Li Ion) battery cell voltage models for general Electric Power System (EPS) simulation and modeling applications. Dependent on this is the appropriate derivation of the requirements and parameters needed to perform the modeling. Also inherent in the project is Model Based System Engineering (MBSE), where modeling is intended to foster design of the total system and evaluate specific performance response of the sytem to the mission. The MBSE concept allows rapid and relatively inexpensive evaluation of the system. The modeling presented in this project accomplishes this, by providing a detailed component model to the higher system level model to evaluate and refine the total system quickly and efficiently.

Focussing purely on the battery component model for a moment, detailed electrochemical cell and battery models have been documented in the literature, including the method by Isaacson (Ref 1) used in this project. For any given Li Ion battery cell manufacturer, a model can be developed that describes the output voltage of a given battery cell as a function of several input parameters. The model for each cell can be determined from basic physical effects, coded in a computer program, and used to verify or validate the model against data measured from that cell. Given the proper input parameters, results from the models that compare very well to the measured data are said to be verified against the data. When others use the models to compare against additional 
data, if the comparison is accurate, then the models are said to have been validated against that data.

The model has been developed in a general mathematical form, and can be applied in any simulation. Specifically, the model described in this project was written within an Excel workbook as simple function code packages, called Excel Visual Basic Macros. In this form, the models can be called from within any other Excel workbook, just as a sine or cosine function. This enables the battery cell voltage model to be easily used by anyone using the Microsoft Excel application.

The model has been derived from a physical analysis of the operation of a battery cell, where the resulting voltage may depend on a number of factors. The same modeling technique is used to develop battery models for the physical battery cells supplied from multiple cell vendors studied in this project.

The data measured from each battery cell during its operational testing is then analyzed. As the physical make-up of each battery cell from each vendor is different, so are the resulting data from the testing of the various battery cell tests. It is found that only certain tests are required in order to derive the data needed for the construction of this battery cell voltage model.

Next, the resulting model parameters and the model results are plotted against the test data. These results are shown (minus axes values) for a sample vendor, annotated "Vendor 1 ".

Finally, suggestions for future work are summarized. 


\section{Prerequisites}

In general, the model presented herein can apply to any number of systems including terrestrial, air, sea or space. The particular detailed application presented for this project is space, and more specifically, satellites. Prior to applying the model, the user must have been delivered the main battery requirements. It is assumed that the higher level system requirements have been defined, in at least a basic form, so that detailed application of the modeling herein can be applied. A general understanding of the higher level system requirements by the battery modeler is helpful to promote efficient total system refinement. After these parameters have been flowed to the modeling user, detailed mission analysis can be performed.

There are a number of ways that the detailed battery requirements can be derived and provided to the battery modeling user. Most companies or system developers utilize some form of system sizing model. This model generally includes the total system, including power and energy assumptions, the acceptable voltage range, the power system type (regulated/unregulated, etc) and mission duration that could include calendar life and number of charge/discharge cycles. As explained in Ref 2, the detailed parametric studies using the total system model produce the detailed battery requiements. It should be noted that the process is iterative; where the detailed battery modeling often influences the system greatly, and the system and battery is modified according to the refined needs of both. Also, such information needed to perform the detailed total system analysis is not always available for a very immature system. In these cases, a basic system model in the form of a simple excel spreadsheet will suffice with assumptions (including appropriate conservatism) for the important system characteristics noted above and 
needed for the detailed mission modeling. It should also be noted that, although the end goal for the battery model is usually to be part of the total system model, the battery model developed and presented here can be used as a stand-alone application. Detailed battery-only response to the given input parameters and cases can be analyzed, including boundary conditions and sensitivity analysis.

\section{Battery Modeling Approach}

The modeling of the voltage for a LiIon battery cell follows the same modeling theory that has been used in the past for Nickel-Hydrogen battery cells. Traditionally, this theory is known as the "Randles Circuit Model" (Refs 3-7). Application of techniques by Isaacson (Refs 1 and 8) are applied for specific application of Li Ion technology to space systems.

It should be noted that much more complicated physics based models have been developed (Refs 8 and 9) but for computational efficiency this work uses the simplest form for the model that will still fill the requirements for the higher level power system modeling and simulation. For this reason the more subtle effects and components of the more complicated model are neglected, including those listed below.

In this model, the battery cell is modeled as a voltage source whose internal resistance is modeled by a resistance (or impedance) followed in series by a resistor and a capacitor which are in parallel. By analyzing certain test data, the values of these electrical components can be determined, which have proven to accurately represent the measured data. 
For the purposes of this project, the time-dependence of the capacitance effect in this impedance model is ignored, and the model consists of only a resistive voltage drop within the cell. For practical use of this model for most satellite applications, the discharge durations are relatively long, on the order of minutes to an hour, where the capacitance effects should diminish within seconds of application of a sustained load.

When using this model, two attributes of the battery cell operation become apparent: (1) that the voltage of the cell changes in a linear manner with the current during charging or discharging, and (2) that the voltage of the cell changes also in a roughly linear manner with the amount of capacity in the battery cell that can be better defined by a fairly complicated open-circuit voltage curve.

Thus, knowing the characteristics of these effects, a voltage model for the cell can be determined.

\section{Open Circuit Voltage Curve}

The Open Circuit Voltage Curve is defined as the voltage measured during an open circuit, or at zero current, as a function of the capacity of the battery. Thus, we can measure

$$
\operatorname{Vocv}=\operatorname{Vocv}(S O C)
$$

where Vocv is the Open Circuit Voltage and SOC is the State of Charge of the battery at any given time (given either in Ah or as a \%). This relationship is usually linear in form, varying over the SOC range, usually from $0 \%$ to $100 \%$, or from 0 Ah to the maximum full or operational capacity of the battery in Ah. 


\section{Cell Impedance Effects}

During discharge, the battery cell voltage will drop during a rise in current due to the impedance effects within the cell, modeled by the resistance in the Randles Model. Similarly, the voltage will rise when the current is reduced. The opposite applies in the charging operation. Using rapid step changes in the current from a zero current condition, the amount of the voltage change can be measured, and these impedances effects can be calculated. It has been observed that this calculated impedance effect may be different for charging and discharging situations, and different values for each may be assigned as appropriate in the model.

Given that Vocv is known at a given State of Charge, the cell impendence (dV/dI), derived from Ohms Law $(\mathrm{V}=\mathrm{I} * \mathrm{R})$, can be calculated by

$$
\mathrm{dVdI}=(\text { Vocv }- \text { Vmeasured }) /(\text { Current Change from Zero Current })
$$

For this model, an impedance effect is calculated for charging pulses, and a separate impedance effect is calculated for discharging pulses. Thus:

$$
\begin{aligned}
& \text { Vchg }=\text { Vocv }+ \text { dvdi_chg } * \text { Current_Pulse_Charging_From_Zero } \\
& \text { Vdis = Vocv }+ \text { dvdi_dis } * \text { Current_Pulse_Discharging_From_Zero }
\end{aligned}
$$

The impedance may also depend on the size of the current pulse, or the size of the change of the current over a specified time. At present, these effects are ignored. The impedance effects are also calculated using the highest charging and discharging current pulses in order that the entire range of current pulses can be included in the model, as accurately as possible. 
It should be noted that the current change does not have to change from zero to some non-zero value. Equally relevant are changes from one non-zero discharge rate to another and the same equations apply.

\section{Model Input Data Summary}

The Li ion battery cell voltage model thus depends on the following measured and calculated parameters:

Model Input Data

To $\quad$ The temperature of the data measured in degrees $\mathrm{C}$ SOC State of Charge points where Vocv is measured

Vocv(SOC) The Open Circuit Voltage measured at the SOC points dvdi_chg Impedance effect during charging dvdi_dis Impedance effect during discharging dvdt Temperature effect

\section{Model Limitations}

\section{Capacitance Effects}

The amount of capacity in the battery cell may change the Open Circuit Voltage curve, $\operatorname{Vocv}(\mathrm{SOC})$, from a rigid linear relationship, and this effect has been seen to be very small.

A more pronounced effect of the cell capacity may be its affect on the cell impedance, as the cell capacity changes. This effect is presently not included in the model.

\section{Temperature Effects}

The change of the voltage at any specified SOC and current pulse condition may be very difficult to mathematically simulate, due to the physical construction of the battery cell. The model includes the ability to measure the Open Circuit Voltage curve at 
any set of given temperatures, and then to calculate from the data the impedance effects for the same bounding charging and discharging current pulses. Know these data, the model can then accurately predict the voltage of the cell at any of those given temperatures, and then can linearly interpolate the data for any temperature between the given temperature points.

The model then includes a temperature effect (dvdt) that varies in a linear manner with the voltage:

$$
\text { Vcell }=\text { Vcell }(\text { To })+\text { dvdt } *(\text { TempC }- \text { To })
$$

where Vcell(To) is the voltage measured at temperature 'To' degrees C, and Vcell is the desired temperature to be calculated at temperature 'TempC' degrees C.

Although temperature effects are included in the code for the modeling, the assigned constants (and thus effects from temperature) are recommended to be set to 20 to provide no influence on the modeled voltage. Use of temperature effects may be included and recommended at a later date, as temperature characterization of the cell is performed. It is important to note that the temperature dependence on the OCV curve is very small for the lithium ion batteries modeled for this project, and the model was derived for temperatures at approximately 20C.

\section{Time-Dependent Effects}

Test data show that the rise and fall of the voltage after a rapid change in the charging or discharging current will not occur instantaneously, and will instead be slow and smooth. This relaxation effect is modeled by the capacitance in the original Randles Model, which is ignored in this model. However, this effect can easily be added by 
assuming that a specified fraction of the voltage is allowed to be an instantaneous change while the remainder is modified by an exponential relaxation coefficient, to account for the change with respect to time.

For the purposes of this project, this effect of the voltage relaxation and timedependence is not included. It is noted that from the standpoint of the system analysis, not including the time-dependent effects is typically conservative, producing predicted voltages higher on charge and lower on discharge at the start of the current transitions. Still, based on comparison to the data, this inaccuracy has a minimal effect on the system analysis and predicted margins.

Results of this model with time-dependence may be released at a later time.

\section{Data Sources for Verification and Validation}

Various Lockheed Martin cell test data were used to develop and verify the battery voltage model. In these tests a variety of $\mathrm{Li}$ ion cells from multiple cell vendors are being tested and a vendor specific model has been developed for each. The following list shows the tests of interest for this project that relate to the modeling of the $\mathrm{Li}$ ion battery cells:

\begin{tabular}{ll}
\multicolumn{2}{c}{ LiIon Battery Cell Tests } \\
COED & Charge Off End of Day \\
COER & Charge Off Every Rev \\
FDAC & Full Discharge and Charge
\end{tabular}

The 'COED' test consists of a variety of cycles of various depths of discharge throughout a $24 \mathrm{hr}$ period. This profile allows characterization of the cell voltage over 
multiple charge and discharge currents with relatively fast transitions from charge to discharge. Such a profile might be experienced in a "Low-Earth" or "LEO” type of orbit.

The 'COER' test consists of multiple back-to-back, relatively high depths of discharge and fast transitions from charge to discharge. This is also a profile that might be experienced in a LEO type of orbit.

The 'FDAC' test consists of a full discharge and recharge of a cell, to characterize the cell voltage over the entire SOC range. This profile would be relevant to any discharge that is prolonged and reduces the cell SOC to low values, including zero. This is a profile that might be applicable to acceptance, qualification or other check-out testing, or “Geostationary or Geosynchronous Earth” or “GEO” and "Medium Earth” or “MEO” type orbits.

Data is taken during these tests and recorded in large data files for later analysis. The data measured include the year, day, and time of each test measurement, as well as the current, voltage, and temperature of each cell, and several other output parameters. These data are stored in tab delimited text formatted data files, where each file contains the data for one day of testing. These data files are typically 2,800 to 3,000 lines long, and are each about $1 \mathrm{Mb}$ in size. Each row contains the data taken at the stated time. The size of the time steps between the data rows can vary, but is usually on the order of 30 seconds.

The test data in these files are used to determine the data that is input to the various battery cell voltage models. The test data is formatted in the test data files, in 
text only format, in delimited columns that is easily imported into MS Excel. It includes current, temperature, voltage, accumulated Ahrs for the phase, date, elapsed time, etc.

It is noted that the test data itself does not include state-of-charge or depth-of-discharge values. These variables can be calculated from the exiting data in each data row by integrating the current and comparing the calculated capacity of the cell to an assumed “full”, or $100 \%$ SOC, capacity value.

The modeled (predicted) cell voltage is calculated using a coded subroutine, rather than by using cell formulas. This simplifies the calculation process and reduces complex problems with Excel whenever errors occur in the model data or the formulas. The coded subroutine then reads from each row, one row at a time, the input data variables, calls the model's function routine to calculate the cell voltage, and copies the result into the output cell in that same row. In this way the model results can easily be plotted against the data as a function of time over any time period within the domain of the test data.

\section{Model Development and Results}

The model development and validation results are presented using a single vendor test results, as an example of the model accuracy, although models have been generated and are in use for each vendor in test. The prerequisite battery requirements (capacity, voltage, etc) have been delivered to the battery modeler and the test data has been analyzed using the model to derive the variable coefficients needed to model the battery for this particular application. 
The model is written in an Excel workbook, using Excel Visual Basic Macros.

These macros are essentially written in the same code format as FORTRAN or C codes and are very easy to use, access, read, understand, and change if necessary.

The model is written as the code function:

Function BattPointsModel_V(soc, curr, temp)

This function can be placed into any Excel workbook.

The function uses the following inputs as variables:

soc State of charge of the cell in percent

curr Current into or out of the cell in Amps

+ for charging, and - for discharging

temp Temperature of the cell in Degrees C

The input variables are not changed by the call to the function.

These variables are read by a subroutine testing code that reads these variables from an input spreadsheet and places the calculated results into the sheet for plotting. The subroutine reads all of the input variable values from the spreadsheet named "Batt Points Model Data”. This sheet contains all of the data that is required to execute the battery cell voltage model. An example of the data input variables worksheet format is included in the Appendix.

In summary, the model consists of three parts: the function code, the subroutine testing code, and the input data worksheet. The model can be used in any Excel workbook that includes these three items. 


\section{Vendor 1 Open Circuit Voltage Curve and Other Parameters}

The data that comprises the Open Circuit Voltage (Vocv) data curve that is currently used in this model is not shown due to its potential proprietary nature, however, sample OCV curve data is shown in the table below and plotted in Figure 1. This provides an understanding of the form and format of the curve used in the model.

\begin{tabular}{l} 
Sample LiIon Model Vocv Cur \\
$\qquad$\begin{tabular}{|c|c|}
\hline SOC & V Cell \\
\hline 110 & 4.20 \\
\hline 105 & 4.15 \\
\hline 100 & 4.10 \\
\hline 95 & 4.05 \\
\hline 90 & 4.00 \\
\hline 85 & 3.96 \\
\hline 80 & 3.94 \\
\hline 75 & 3.91 \\
\hline 70 & 3.88 \\
\hline 65 & 3.86 \\
\hline 60 & 3.84 \\
\hline 55 & 3.83 \\
\hline 50 & 3.81 \\
\hline 45 & 3.80 \\
\hline 40 & 3.78 \\
\hline 35 & 3.75 \\
\hline 30 & 3.72 \\
\hline 25 & 3.71 \\
\hline 20 & 3.65 \\
\hline 15 & 3.59 \\
\hline 10 & 3.48 \\
\hline 5 & 3.10 \\
\hline 0 & 2.00 \\
\hline & \\
\hline &
\end{tabular} \\
\hline 50 \\
\hline 50
\end{tabular}




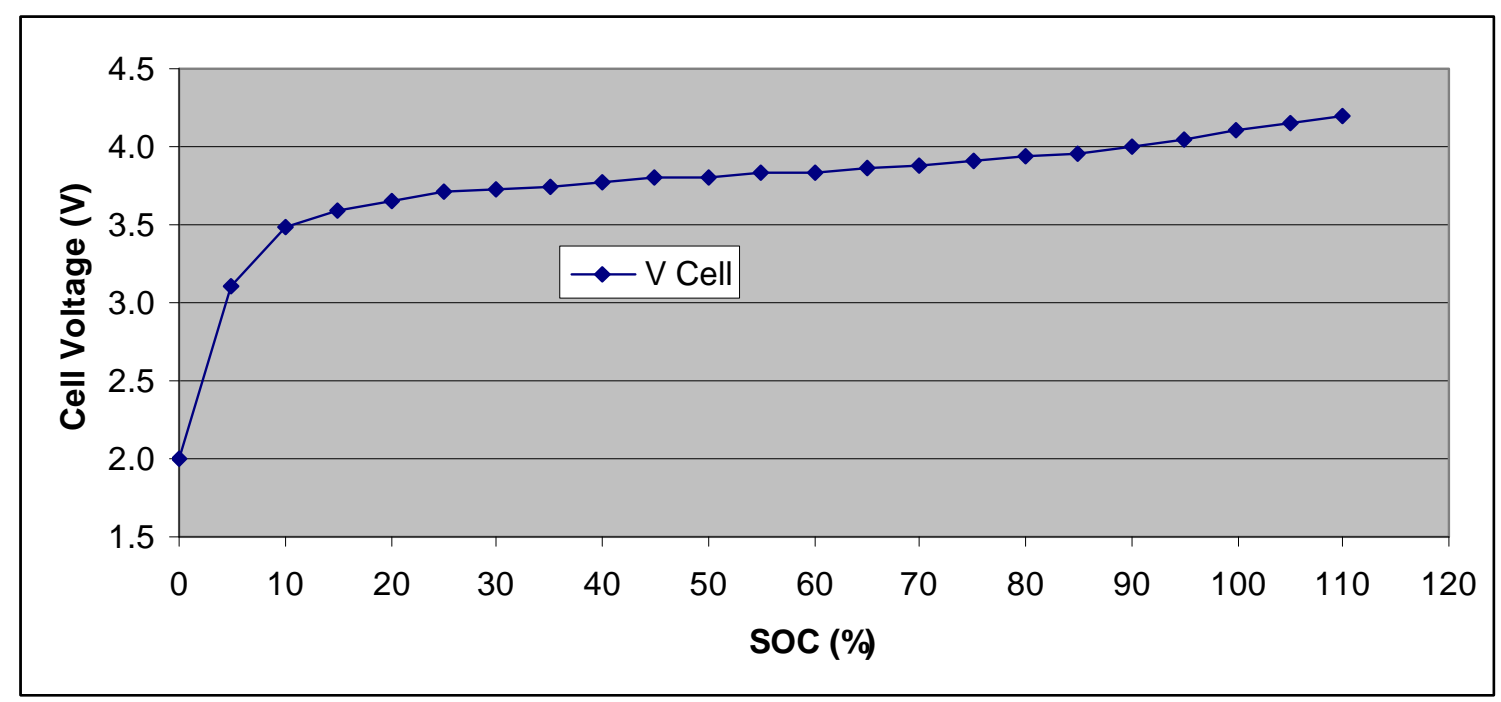

Figure 1. Sample Li ion Model Vocv Curve

It is noted that these data do not follow a strict linear relationship to the SOC. Also, note that SOC values greater than $100 \%$ may occur if the cell is rated lower than the actual capacity it can deliver.

The Vocv data curve is input into the model using an input data spreadsheet described below. The number of data points and the values in these data can always be changed in the spreadsheet.

The value of the model input variables are determined by comparing the testing data to the model for various cases, and adjusting the parameters to achieve the best model fit to the data. The resulting model parameters for this model are given below:

$\begin{array}{ll}\text { Example Vendor LiIon Mode } & \\ \text { To } & 20.0 \\ \text { dvdi_chg } & 0.0013 \\ \text { dvdi_dis } & 0.0013 \\ \text { dvdt } & 0\end{array}$

These values are included in the input data spreadsheet. 
In addition to adjusting these parameters, a normalization of the capacity in the spreadsheet to match the relative SOC is performed.

It is important to note that, given the above modifications, the voltage model matches the test data very accurately, within the needs of the higher level system modeling. The end result achieved is a model that is verified against the test data with good correlation.

\section{Vendor 1 Voltage Model Results}

The results of using these model parameters in this model are shown in the

following figures. In each case the model results are plotted against the actual test data as measured and recorded in the test data files. Excellent correlation is observed with only minor differences during the "taper" charge regions and during the full charge of the FDAC test.

It is noted that all of the data and model results are taken at 20-23C, similar to planned use, except the discharge in the FDAC test where the cells can warm to as high as $30 \mathrm{C}$ at the end of the discharge. Fortunately, although the temperature effect is included but not used in the model, the impact of temperature on $\mathrm{Li}$ ion cell voltage is usually relatively small and the predicted voltage overlays with the test data accurately even at the higher temperatures. 


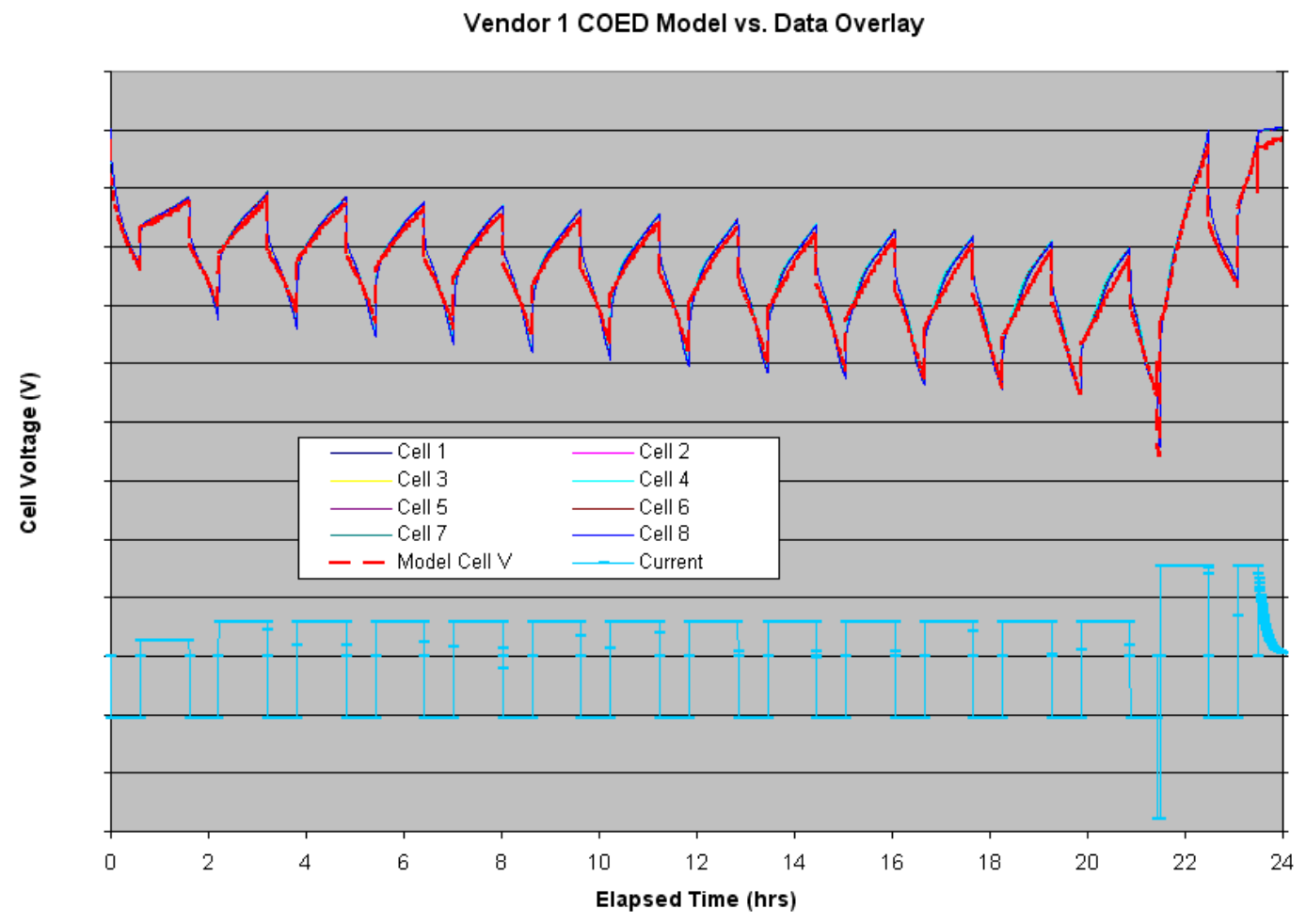

Figure 2. COED Results

Vendor 1 COER Model vs. Data Overlay

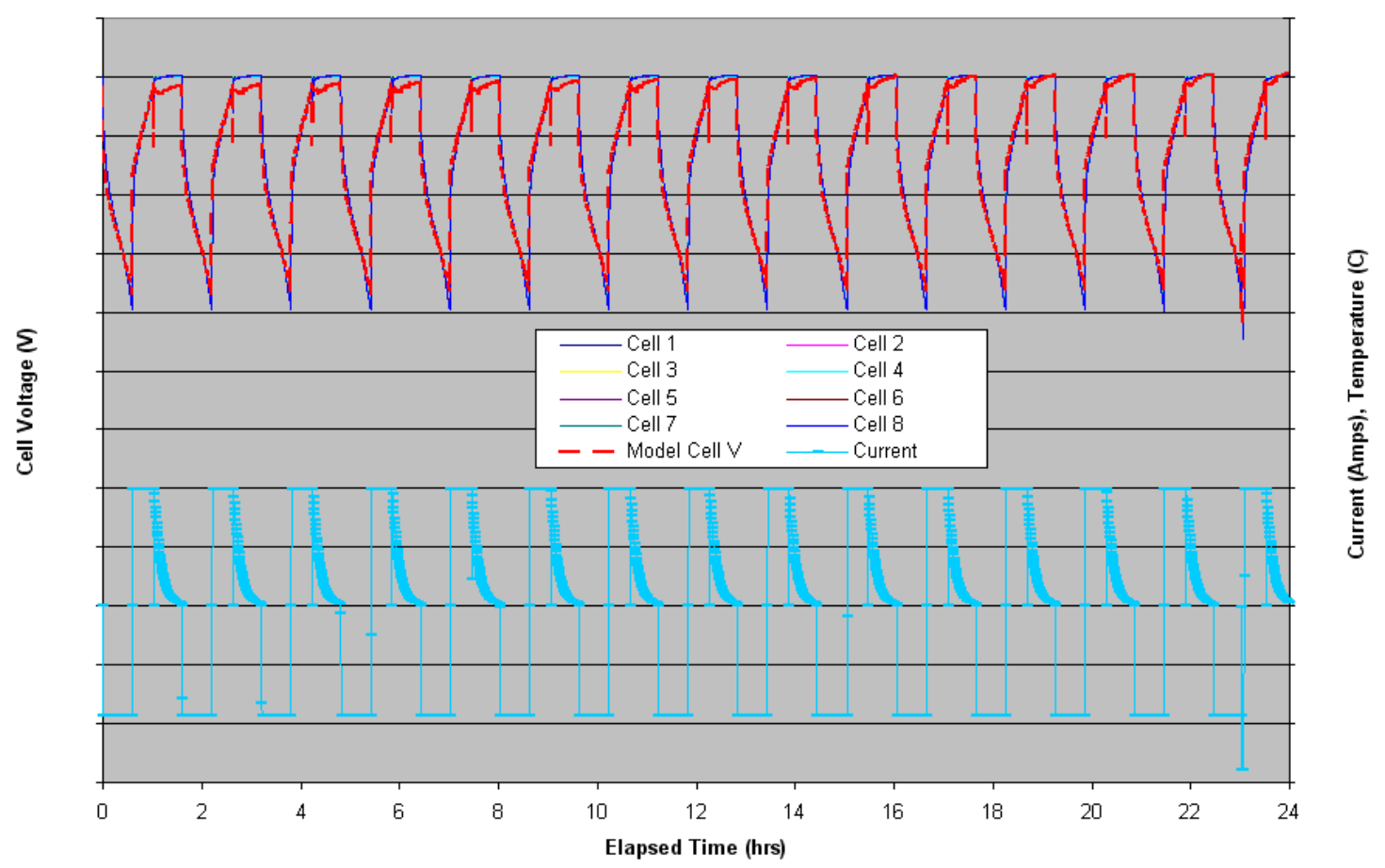

Figure 3. COER Results 


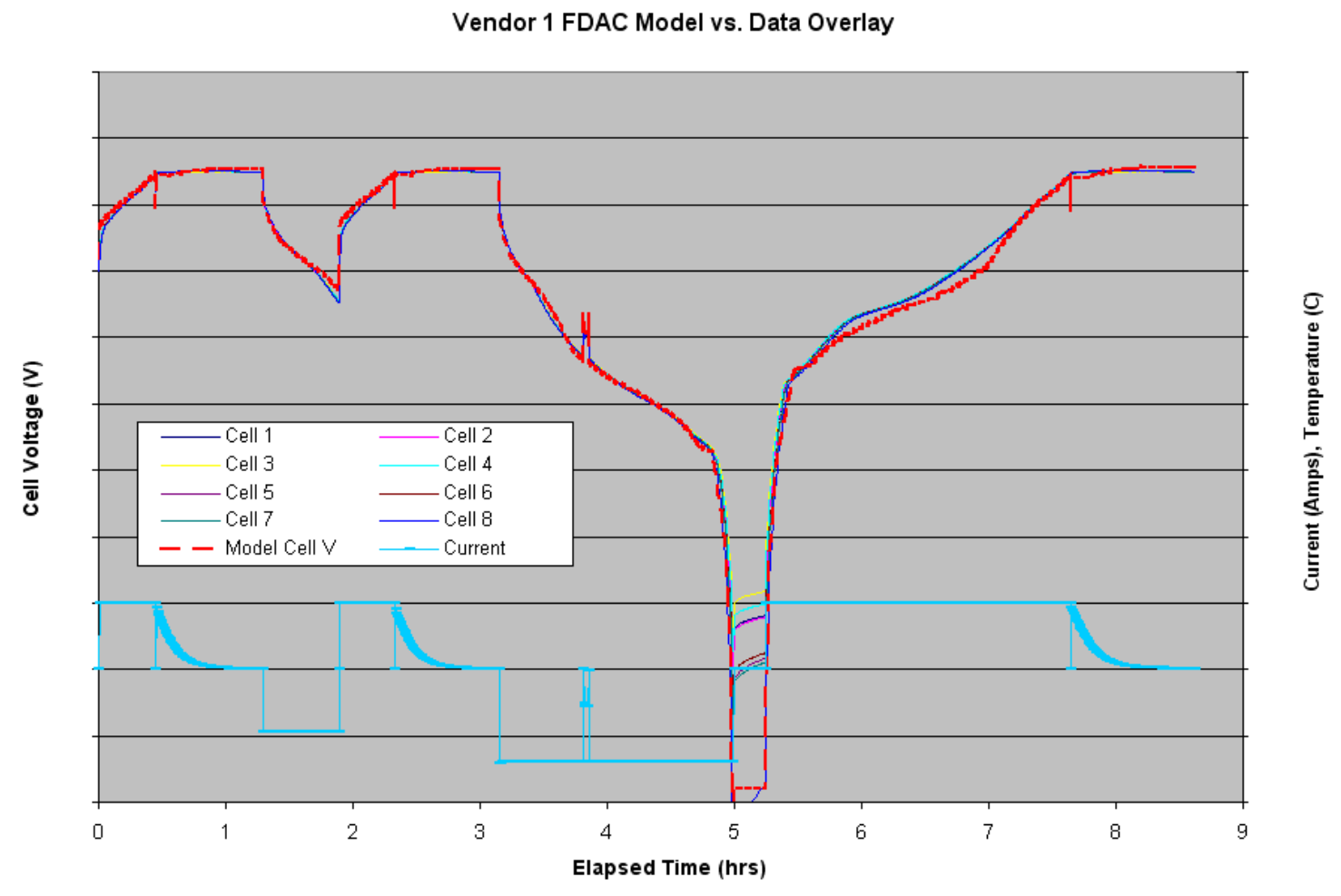

Figure 4. FDAC Results

\section{Future Work}

Future work will include refinements to the models to more accurately model the taper charge region. Providing additional battery models as new assets are tested will also be included. As life test data is gathered, end-of-life projections will be implemented in the models to predict cell voltages for specified mission durations.

\section{Project summary}

This project describes a detailed battery cell voltage model used to size and predict performance of complex total systems using Model Based System Engineering. The general approach to developing and implementing the model used system engineering principles to provide prediction capability for all aspects of the mission. The necessary baseline requirements for the battery have been generated and flowed down to 
the battery modeler, and the appropriate battery model parameters have been obtained from applicable test data. The end product is an accurate and versatile battery model that easily interfaces with the higher detailed total system model to refine and optimize the total system and predict performance and margin. The models presented are in use and have resulted in more efficient, lower risk systems due to the ability to predict performance with high fidelity in nearly all operational scenarios.

\section{References}

1. "Physics-Based Modeling of a 40 Ah Li-ion Battery for Geosynchronous Earth Orbit (GEO) Satellite Applications," Mark J. Isaacson and Robert Spotnitz, $220^{\text {th }}$ Electrochemical Society Meeting and Electrochemical Energy Summit, October 9-14, 2011.

2. Power Tools Suite (PTS) Models and Codes, Volumes 1-6, Lockheed Martin Proprietary software and documentation, 2011.

3. "Electro-thermal modeling of polymer lithium batteries for starting period and pulse power,” Journal of Power Sources, Vol 54, pp 393-396, 1995.

4. "Battery Modeling for In-Situ Prognostics," D. S. Bodden and N. S. Clements, Joint TFG Conference, Lockheed Martin Advanced Technology Center, April 57, 2011.

5. "Dynamic Lithium-Ion Battery Model for System Simulation," Lijun Gao, Shengyi Liu, and Roger A. Dougal, IEEE TRANSACTIONS ON COMPONENTS AND PACKAGING TECHNOLOGIES, VOL. 25, NO. 3, SEPTEMBER 2002, pp 495-505.

6. "New battery Model and State-of-Health Determination Through Subspace Parameter Estimation and State-Observer Techniques,” C. R. Gould, C. M. Bingham, D. A. Stone, and P. Bently, IEEE Transactions on Vehicular Technology, Vol. 58, No. 8, October 2009, pp 3905-3916.

7. "Lithium-Ion Battery Modeling Using Constant Load Data," N. Scott Clements, David S. Bodden, Lockheed Martin Internal Report, May 2011.

8. Abstract \#752, Mark J. Isaacson, 220th Meeting of The Electrochemical Society, Oct 9-14, 2001, Boston MA

9. M. Doyle, J.P. Myers and John Newman, J. Electrochem. Soc, 147(1), 99-110 (2000). 


\section{Appendix}

The code listing of the function for the battery cell voltage model is given below.

The format of the input values spreadsheet is shown in the next sub-section.

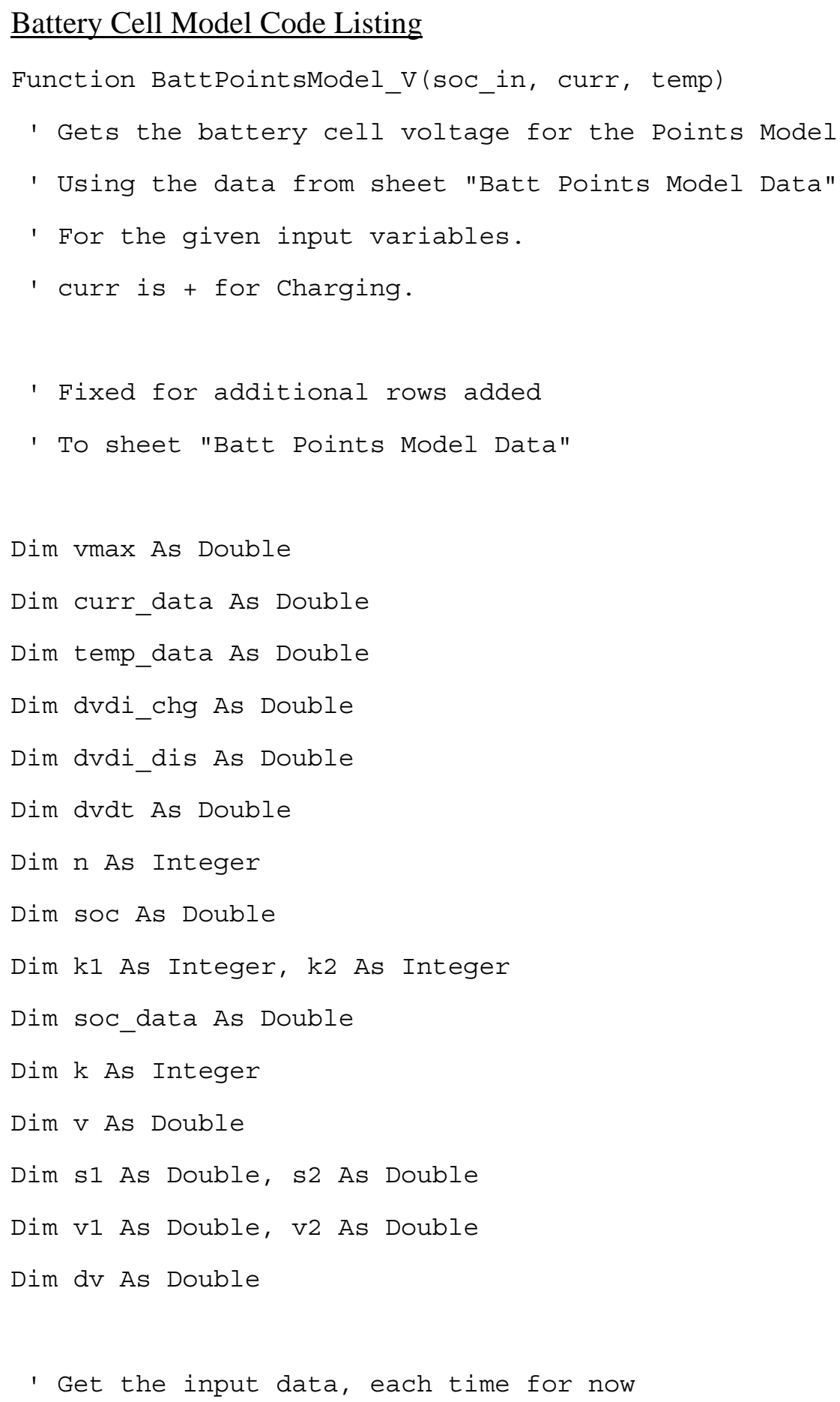




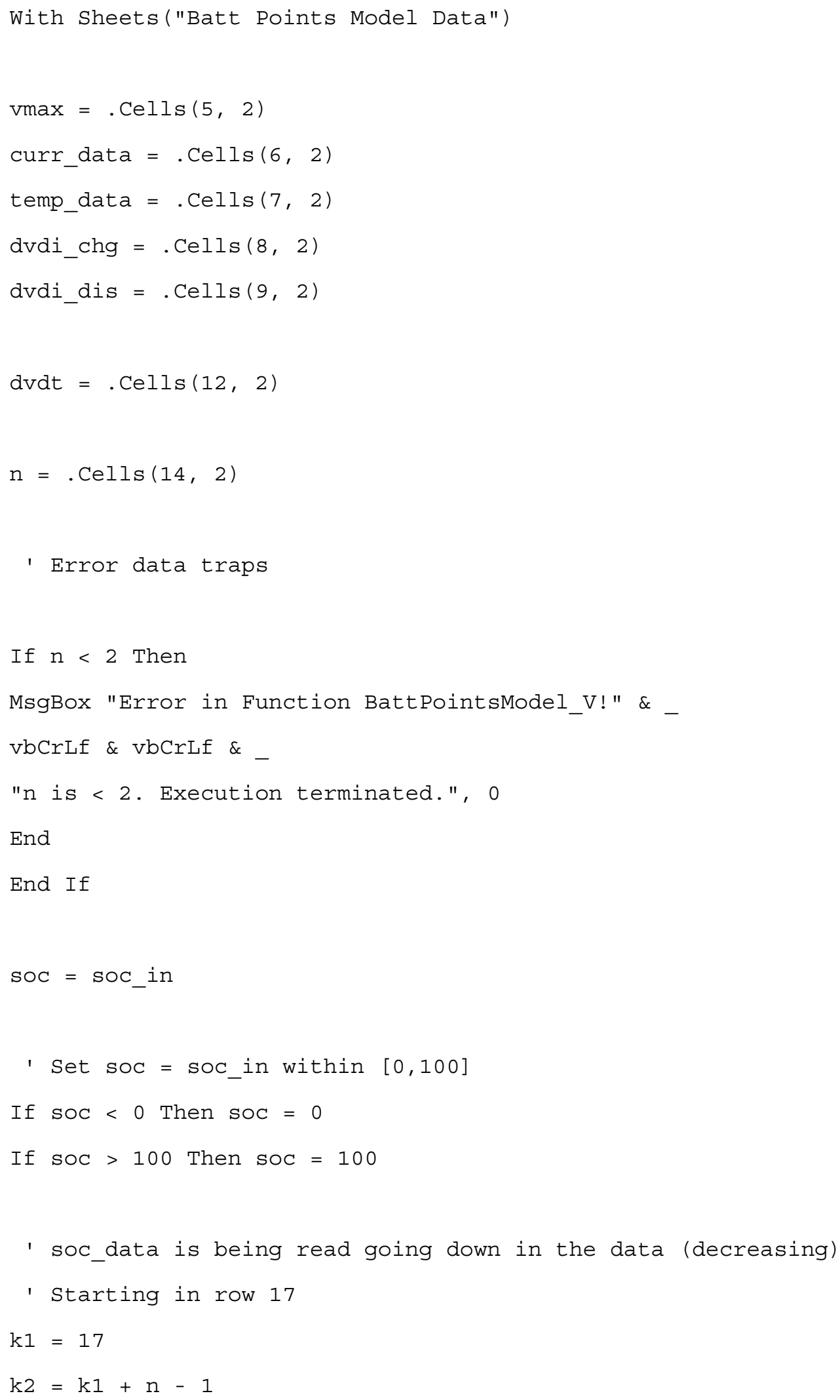




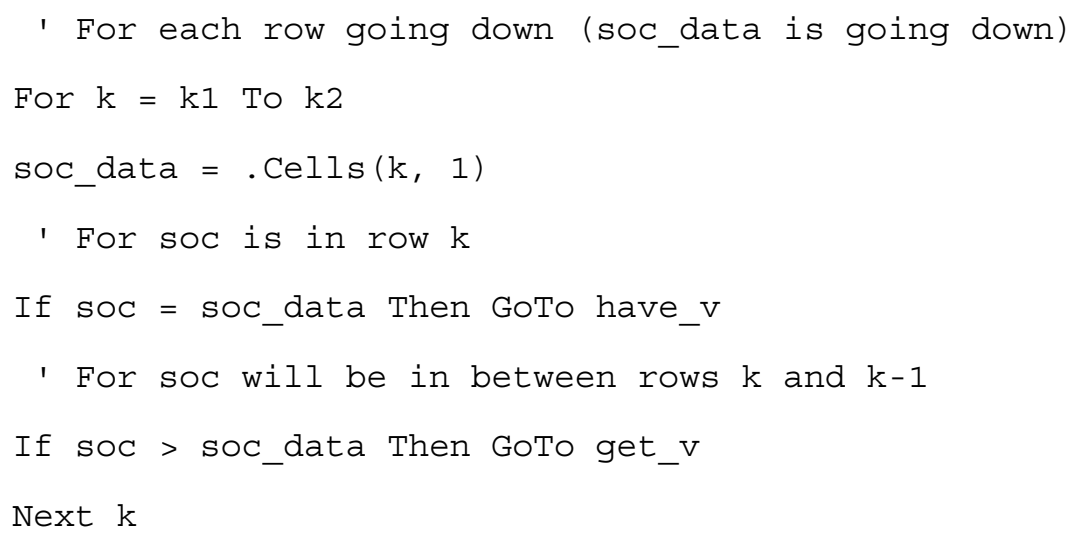




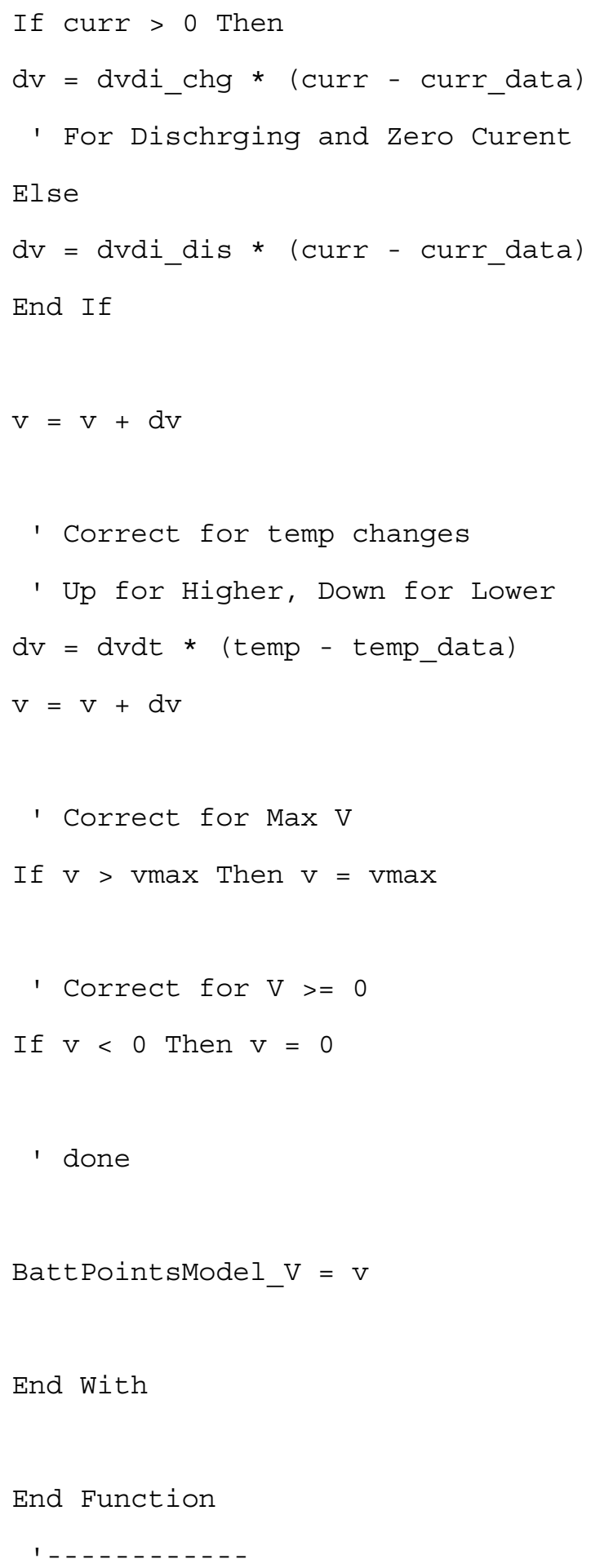

Battery Cell Model Input Variables Data Worksheet

A sample data spreadsheet with the name "Batt Points Model Data" is given in the following figure to show how the input variables are given to this model. 
The input data given in column B defines the model being used. The input variables shown are self explanatory. The variable "NPoints" defines the number of points that are to be read in as the $\operatorname{Vocv}(\mathrm{SOC})$ curve in the cells below.

Note that the name of the spreadsheet and the cell locations of these data must be exactly as described for the model code listed in the previous section to work properly.

\begin{tabular}{|c|c|c|c|}
\hline & A & $\mathrm{B}$ & C \\
\hline 1 & Nameplate & 100 & Ah \\
\hline 2 & SOC Initial & 100 & $\%$ \\
\hline 3 & Vmax & 5.00 & (1 for use points) \\
\hline 4 & IData & 0 & At Amps for the Data Given \\
\hline 5 & T Data & 20 & At Deg. C for the Data Given \\
\hline 6 & DVilDI_chg & 0.00130 & Change of Voltage per Amp Chg \\
\hline 7 & DW'Dl_dis & 0.00130 & Change of Voltage per Amp Dis \\
\hline 8 & D'VIDT & 0.00000 & Change of Voltage per Deg. C \\
\hline \multicolumn{4}{|l|}{9} \\
\hline 10 & N Points & 11 & The number of data rows below \\
\hline \multicolumn{4}{|l|}{11} \\
\hline 12 & $\mathrm{SOC}$ & V Cell & \\
\hline 13 & 100.00 & 4.10 & \\
\hline 14 & 90.00 & 4.00 & \\
\hline 15 & 80.00 & 3.90 & \\
\hline 16 & 70.00 & 3.80 & \\
\hline 17 & 60.00 & 3.70 & \\
\hline 18 & 50.00 & 3.60 & \\
\hline 19 & 40.00 & 3.50 & \\
\hline 20 & 30.00 & 3.40 & \\
\hline 21 & 20.00 & 3.30 & \\
\hline 22 & 10.00 & 3.20 & \\
\hline 23 & 0.00 & 3.10 & \\
\hline
\end{tabular}

Figure 5. Sample Battery Cell Model Code Input Variable Data Spreadsheet 